\title{
The Effect of First-Step Techniques from the Staggered Stance in American Football
}

\section{(ㄷ) (1) (\$) $\Theta$}

Authors

Nikolas Sten Knudsen, Thomas Bull Andersen

Affiliation

Aarhus Universitet, Department of Public Health - Sport Science, Aarhus, Denmark

Key words

chaos sports, running, acceleration, push-off

received 01.09 .2016

revised 19.01.2017

accepted 25.01.2017

Bibliography

DOI http://dx.doi.org/10.1055/s-0043-103010

Sports Medicine International Open 2017; 1: E69-E73

(c) Georg Thieme Verlag KG Stuttgart · New York

ISSN 2367-1890

\section{Correspondence}

Nikolas Sten Knudsen, Cand.scient.

Aarhus Universitet

Department of Public Health - Sport Science

Dalgas Avenue 4,

8000 , Aarhus

Denmark

Tel.: +45/602/40 579

nikolas_35@hotmail.com

\begin{abstract}
The purpose of this study was to evaluate 3 different starting techniques from the staggered stance with regards to sprint time, reaction time, linear impulse and power. 11 male amateur American football players volunteered to participate in a testing session consisting of twelve $5 \mathrm{~m}$ sprints, 4 in each technique (normal (NORM), backwards false step (BFS) and forwards false step (FFS)) in random order. Sprint starts were performed on force plates to investigate ground reaction forces, reaction time and total sprint time. Analysis showed significant differences in sprint times, with NORM $(1.77 \pm 0.10 \mathrm{~s})$ being faster than FFS $(1.81 \pm 0.12 \mathrm{~s})$ and BFS $(2.01 \pm 0.13 \mathrm{~s})$, and FFS being faster than BFS, although no differences were found in reaction time. In terms of mean force and power, NORM $(331.1 \pm 39.2 \mathrm{~N}, 542.2 \pm 72.3 \mathrm{~W})$ and FFS $(320.8 \pm 43.2 \mathrm{~N}, 550.9 \pm 81.4 \mathrm{~W})$ were significantly larger than BFS $(256.9 \pm 36.2 \mathrm{~N}, 443.5 \pm 61.1 \mathrm{~W})$. This indicates that when starting from a staggered stance, the BFS is inferior to the others and should be avoided. However, since the force profiles of the NORM and the FFS were similar, the differences in sprint time could arise from a technique bias towards the NORM start.
\end{abstract}

\section{Introduction}

In many team sports, the ability to change direction and accelerate over short distances is of great importance and has been the focus of many investigations $[3,8]$. For instance, in American football the wide receivers start their run from a staggered stance and must beat their direct opponent by making open field moves or simply outrunning the defender. For such players the acceleration in the beginning of their route needs to be maximized to a) outrun their opponent in a straight-line route or b) get to an open space earlier or at greater depth to optimize the efficiency of the offence [14].

In order to accelerate the body of an athlete efficiently from the parallel stance, the point of impact with the ground must be behind the centre of mass of the athlete [12]. This can be done by either tilting the body forwards before stepping or stepping back with one foot in order to provide the same tilting. Research has shown that the counter-intuitive backwards false step from the parallel stance is a more efficient first-step sprint technique than a normal tilting start in terms of faster $5 \mathrm{~m}$ sprint times [4-6], faster time to peak force [6], higher mean power $[2,12]$ and higher force production [12] possibly due to utilization of the known effects of stretch shortening cycles (SSC) on force production and velocity [11]. However, including the clean start from a staggered stance, the 2 starting techniques provided similar kinetic results [6].

Including the staggered backwards false step (BFS), where the athlete brings the front foot back before stepping through with the back foot, one study found this technique significantly faster than the parallel false step but not different from the normal staggered start [10]. This study was, however, completed without measuring ground contact forces, and thus does not provide possible changes in time to peak force, peak force or power in order to explain the results.

Overall these findings point to the SSC being utilized during the sprint start to provide greater sprint acceleration $[7,11,12]$. 
Because a main skill in American football is to "get off the ball" and maximize initial running acceleration [1], more focus should be put into the get-off phase because subsequent actions are influenced by the quality of the get-off and initial running phase. Indeed, sprint performances in 40-yard sprints are shown to be highly correlated to 5-yard sprint performances [15]. Hence, for wide receivers to be able to outrun their direct opponent, focusing on the get-off phase could prove to be beneficial [1].

In a sport where inches are crucial, if one can get the same beneficial SSC from taking a quick forward false step (FFS) before stepping through with the back foot, it would seem to be a more efficient getoff technique than the backwards false step because the player already has forward momentum before utilizing the SSC and thus obtaining greater get-off speed. To our knowledge, no study has investigated the forward false step from the staggered stance and its implication on initial acceleration and momentum during the get-off phase.

The aim of this study is thus to compare the first-step techniques in the get-off from the staggered stance (NORM, BFS and FFS) with regards to reaction time, time to peak force, generated linear impulse and mean power during the get-off and $5 \mathrm{~m}$ sprint times to determine the most efficient first step of the 3 .

\section{Materials \& Methods}

\section{Research design}

The present study was designed to address the question of how different sprint-starting techniques affect first-step ground-reaction kinematics and $5 \mathrm{~m}$ sprinting performance. We used a repeated-measures design with a randomized order of testing to exclude effects of fatigue or habituation.

\section{Subjects}

11 healthy Caucasian male American football players of varying skill levels (1-7 years of practice, however all amateurs) and positions ( 2 offensive guards, 2 tight ends, 3 cornerbacks, 2 safeties, 1 quarterback, and 1 defensive end) volunteered for this study. Criteria for participation was to use the staggered stance either while playing in their primary position, as in the case of TEs and DL, or as a part of special teams, as in the case of OL, DBs and QB. Their age was $23.5 \pm 3.9$ years (mean \pm SD), their height $186.4 \pm 6.8 \mathrm{~cm}$ and their body mass was $89.4 \pm 16.9 \mathrm{~kg}$. In accordance with the local ethics committee and SMIO ethical standards [9], they all gave their informed consent to participate in the study. Testing was done during the first month of offseason.

\section{Procedures}

After a warm-up consisting of 5 min light cycling on an ergometer cycle (MONARK 818E, Monark Exercise AB, Vansbro, Sweden) at approximately $50 \mathrm{~W}$ (about $15 \%$ of maximum minute power) followed by a short dynamic warm-up of the legs inspired by the participants normal football warmup consisting of 10 squats, 10 lunges per leg, 10 straight-legged front foot jumps, and 10 broad jumps, the subjects were instructed verbally in and shown the 3 starting techniques: NORM ( $\triangleright$ Fig. 1a) BFS ( $\triangleright$ Fig. 1b) and FFS ( $\triangleright$ Fig. 1c) and had a test run for each to make sure the test participants had understood the techniques. Some participants needed more test runs to understand the techniques; however, no more than 3 test runs per technique was permitted. The median number of test runs was one for the NORM technique and 2 for the FFS and BFS techniques. The 3 techniques investigated were chosen to compare the FFS technique to the push-off techniques most commonly used by the wide receivers and special teamers.

During testing, a red light bulb was placed in front of the participants and was lit with a random delay and synchronized with the custom-made timing software (LabVIEW 2015, Austin, Texas, USA). The participants were instructed to sprint as fast as possible once the bulb was lit, using the staggered stance and one of the 3 starting techniques, until they cleared a line approximately $1 \mathrm{~m}$ behind the finish line. The finish line was a photo cell (Photoelectric Proximity Switch WT100, SICK, Inc., Minneapolis, Minnesota, USA) placed $5 \mathrm{~m}$ from the tip of the front foot and timing was stopped once the photo cell was broken. As main testing was performed indoors in the Biomechanics Lab on a track consisting of hardwood and laminate, participants wore their normal shoes used for indoor sport activities. At end of the test track, a gate to the surrounding sports arena was opened to ensure plenty of space for slowing down.

At and behind the starting line, 2 force plates (AMTI, Watertown, Massachusetts, USA) measured the ground reaction forces of the first steps ( $>$ Fig. 2). The force plates were spaced to ensure all participants could start from the split stance using their self-chosen feet splits. The participants were instructed to adjust the length of their steps in order to clear the 2 force plates in the first step in the NORM start, the third step in the BFS start and in the second step in the FFS start. Both timing software and force plates were sampled at $1000 \mathrm{~Hz}$.

Each trial started with the participant drawing one of 12 facedown cards from a table with a get-off technique written on it. After confirming the drawn get-off technique, the participant performed a full-effort sprint using that technique. If the sprint was performed with a technique that did not live up the instructed standard or slipping occurred, the sprint was redone, resulting in about $20 \%$ of the sprints being redone. Participants received feedback on their technical performance; however, no further encouragement was given. After each trial, the participant rested for $45 \mathrm{~s}$. This procedure was repeated until 4 successful trials in each of the 3 techniques were recorded. Before analysis, the force plate data were passed through a 2-pass second-order Butterworth filter with a $20 \mathrm{~Hz}$ cut-off frequency and no phase shift in order to filter out errors from instantaneous muscle twitches before sprint start. Further filtering excluded horizontal forces if the relevant vertical forces fell short of $5 \mathrm{~N}$, indicating the force was produced without contact with a participant. The analysis of the resulting horizontal force diagrams ( $\triangleright$ Fig. 3) was performed in MATLAB ${ }^{\circledR}$ (2015a, The MathWorks, Natick, Massachusetts, USA). Note that only the resulting horizontal force was analysed because it was the only component of the force vector that could influence the motion in the horizontal direction. Reaction time (s) was determined as the first time the absolute value of the resulting horizontal forces exceeded a certain force threshold [13], here chosen to be $50 \mathrm{~N}$. The mean horizontal force $(\mathrm{N})$, power $\left(\mathrm{N} \mathrm{m} \mathrm{s}^{-1}\right)$ and final horizontal impulse $(\mathrm{N} \mathrm{s})$ divided by body mass $\left(\mathrm{m} \mathrm{s}^{-1}\right)$, which equals exit speed, were calculated from the moment of the start signal to the moment when participant left the force plates. 
a

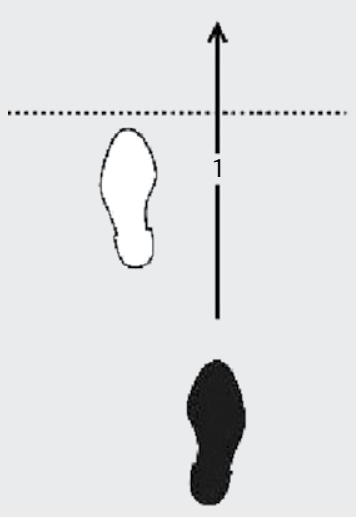

b

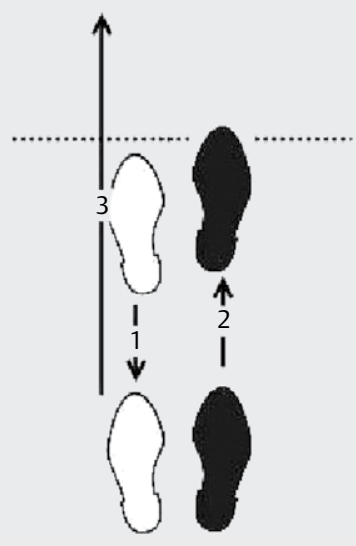

C

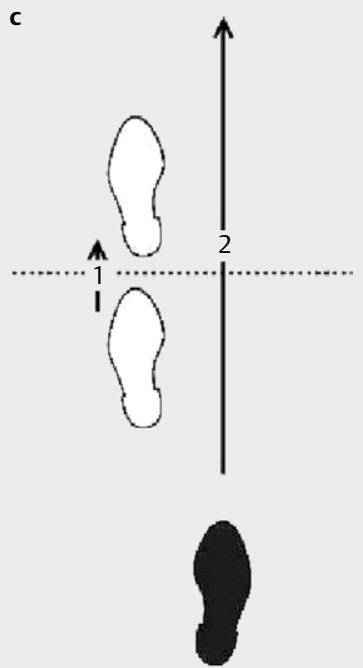

Fig. 1 The tested starting techniques: a NORM, where the first step brings the back foot forward; $\mathbf{b}$ BFS, where the first step brings the front foot backwards; and c FFS, where the first step brings the front foot forwards.

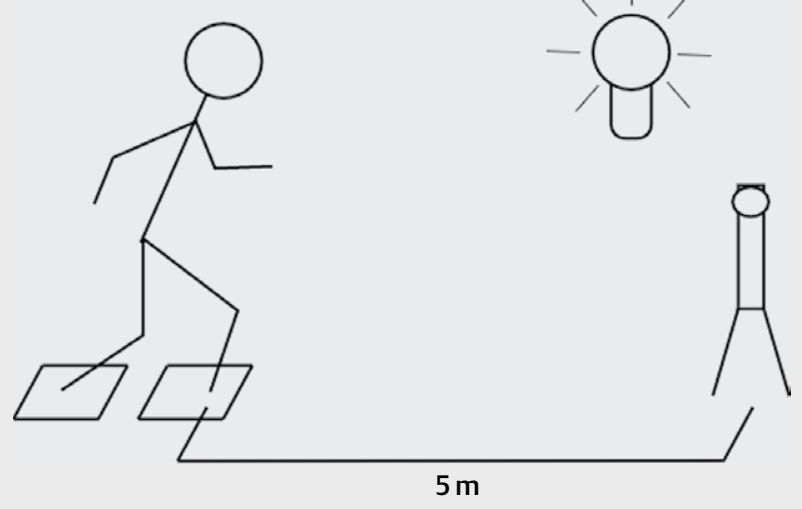

- Fig. 2 Starting position for the sprint starts.

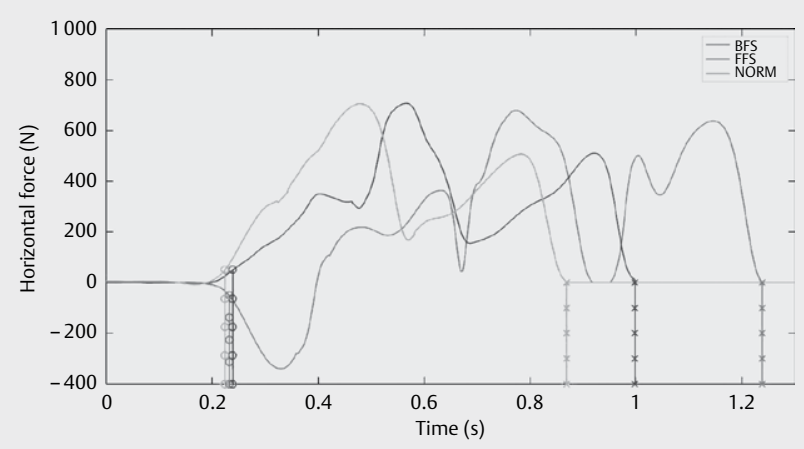

- Fig. 3 Resulting horizontal force - time history for the 3 starting techniques specified in $>$ Fig. 1 . Reaction time is marked by '-o-' and time for leaving the force plates is marked by '-x-'.

\section{Statistical analyses}

Data are presented as means \pm SD. Results from the different starting techniques were statistically compared, using a repeated measures analysis of variance (ANOVA) with a significance level set at $p<0.05$. When significant effects were observed, group differences were found using a Holm-Sidak post-test with the significance level set at $p<0.05$. All statistical analyses were performed using SigmaPlot 12.5 statistical software (Systat Software Inc., San Jose, (A, USA).

\section{Results}

The $5 \mathrm{~m}$ sprint test showed significant differences in sprint times with NORM being the fastest ( $1.77 \mathrm{~s})$, followed by a slightly slower FFS (1.81s) and the slowest BFS $(2.10 s)(p<0.05)$ ( Fig. 4). The

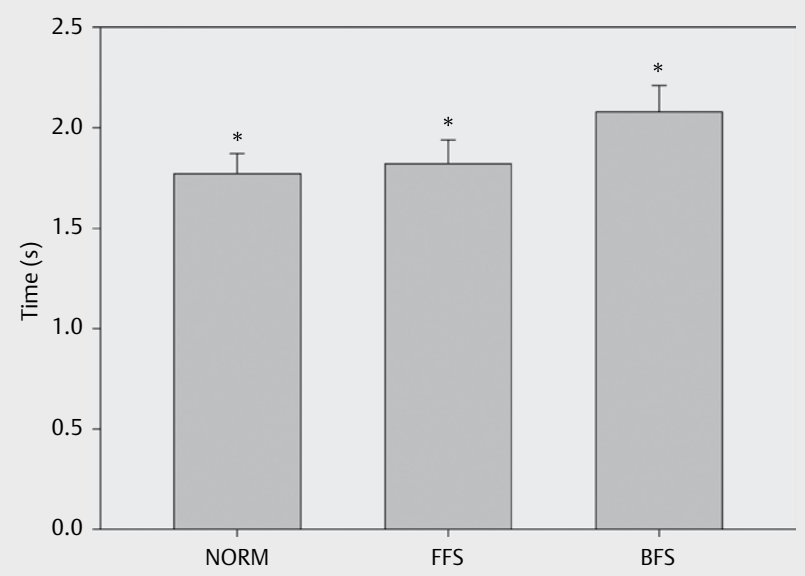

- Fig. 4 Mean $5 \mathrm{~m}$ sprint times for each starting technique $(\mathrm{MEAN} \pm \mathrm{SD}) .{ }^{*}$ Significantly different from all other techniques $(p<0.05)$ 
same significant differences were found in time to peak force, with NORM as the faster $(0.52 \mathrm{~s})$, followed by FFS ( $0.62 \mathrm{~s})$, and BFS as the slowest by quite a margin $(0.95 \mathrm{~s})(\mathrm{p}<0.05)$ (

- Table 1 Time and kinematic characteristics for each starting style $(\mathrm{MEAN} \pm \mathrm{SD})$.

\begin{tabular}{|l|c|c|c|}
\hline & NORM & FFS & BFS \\
\hline $\begin{array}{l}\text { Sprint } \\
\text { Time (s) }\end{array}$ & $1.77 \pm 0.10^{*}$ & $1.81 \pm 0.12^{*}$ & $2.01 \pm 0.13^{*}$ \\
\hline $\begin{array}{l}\text { Time to } \\
\text { Peak } \\
\text { Force (s) }\end{array}$ & $0.52 \pm 0.09^{*}$ & $0.62 \pm 0.15^{*}$ & $0.95 \pm 0.18^{*}$ \\
\hline $\begin{array}{l}\text { Peak } \\
\text { Force } \\
\text { (N) }\end{array}$ & $861.7 \pm 105.5$ & $845.5 \pm 116.5$ & $800.6 \pm 114.6$ \\
\hline $\begin{array}{l}\text { Mean } \\
\text { Force } \\
\text { (N) }\end{array}$ & $331.1 \pm 39.2^{\beta}$ & $320.8 \pm 43.2^{\beta}$ & $256.9 \pm 36.2^{*}$ \\
\hline $\begin{array}{l}\text { Mean } \\
\text { Power } \\
\text { (W) }\end{array}$ & $542.2 \pm 72.3^{\beta}$ & $550.9 \pm 81.4^{\beta}$ & $443.5 \pm 61.1^{*}$ \\
\hline $\begin{array}{l}\text { Time } \\
\text { after }\end{array}$ & $0.89 \pm 0.08$ & $0.87 \pm 0.06$ & $0.88 \pm 0.06$ \\
$\begin{array}{l}\text { Force } \\
\text { Plate (s) }\end{array}$ & & & \\
\hline $\begin{array}{l}\text { Reac- } \\
\text { tion } \\
\text { time (s) }\end{array}$ & $0.23 \pm 0.03$ & $0.25 \pm 0.05$ & $0.25 \pm 0.02$ \\
\hline $\begin{array}{l}\text { Final } \\
\text { Hori- } \\
\text { zontal } \\
\text { Impulse } \\
\text { (m s }{ }^{-1} \text { ) }\end{array}$ & $3.28 \pm 0.31^{*}$ & $3.44 \pm 0.26^{\alpha}$ & $3.46 \pm 0.28^{\alpha}$ \\
\hline $\begin{array}{l}\text { Time on } \\
\text { Force } \\
\text { Plate (s) }\end{array}$ & $0.87 \pm 0.05^{*}$ & $0.95 \pm 0.08^{*}$ & $1.19 \pm 0.11^{*}$ \\
\hline & & & \\
\hline & & & \\
\hline & & & \\
\hline & & & \\
\hline
\end{tabular}

* Significantly different from all other techniques $(p<0.05)$,

$\alpha$ Significantly different from NORM $(p<0.05), \beta$ Significantly different from BFS $(p<0.05)$

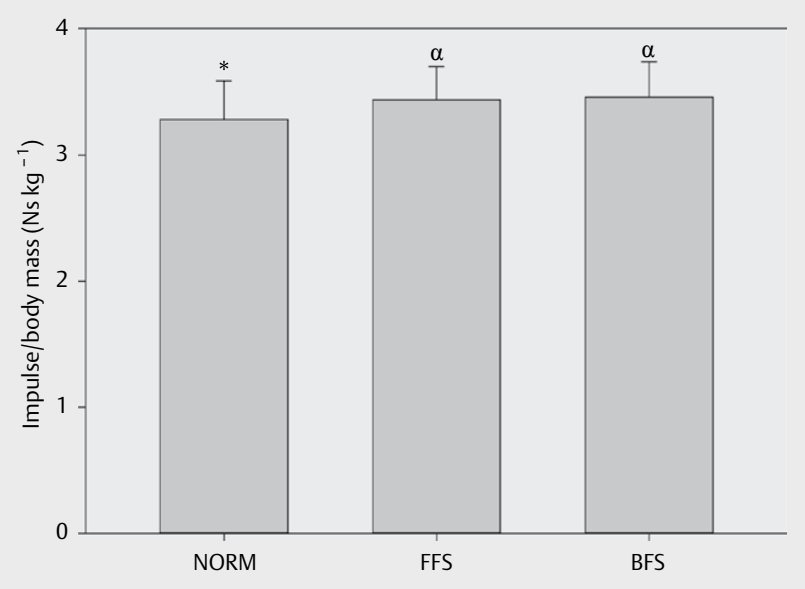

- Fig. 5 Mean horizontal impulse when leaving the plate for each starting technique (MEAN \pm SD). ${ }^{*}$ Significantly different from all other techniques $(p<0.05)$, ${ }^{\alpha}$ Significantly different from NORM $(p<0.05)$.
NORM and FFS showed both significantly higher mean forces (331.1N and $320.8 \mathrm{~N}$ respectively) and power production during the start (542.2W and 550.9W respectively) than BFS $(256.9 \mathrm{~N}$ an 443.5W) (both p<0.05) ( Table 1). Final horizontal impulse was significantly lower when using NORM $\left(3.28 \mathrm{~m} \mathrm{~s}^{-1}\right)$ in comparison to FFS $\left(3.44 \mathrm{~m} \mathrm{~s}^{-1}\right)$ and BFS $\left(3.46 \mathrm{~ms}^{-1}\right)(\mathrm{p}<0.05)$ ( $>$ Fig. 5). Finally, NORM took a significantly shorter time to perform (0.87 s); FFS was slightly slower $(0.95 \mathrm{~s})$ and BFS was the slowest to leave the force plates $(1.19 s)(p<0.05)(\triangleright$ Fig. 6).

\section{Discussion}

The results of this study show that from the staggered stance NORM produces faster $5 \mathrm{~m}$ sprint times in comparison to the FFS and BFS with a sprint time of NORM comparable to similar measurements performed by Frost et al. [7]. Although previous studies have shown improved sprint times using the false step from the parallel stance $[4-6,12]$ and similar times using the backwards false step from the staggered stance in comparison to the normal step [10], once the time spent on the steps and the kinetics of BFS are included, it produces significantly slower $5 \mathrm{~m}$ sprint times than FFS and NORM because the BFS is significantly slower to perform while providing the lowest mean force and power throughout the start. Although the final horizontal impulse of BFS and FFS are not different, FFS provides faster sprint times while spending less time on the force plates. Assuming a similar time-acceleration relationship after the initial get-off phase regardless of technique used in the get-off phase, these findings seem rational because further acceleration after the get-off would be similar in shape but shifted in time. Hence, the more time used initially to obtain similar exit speeds, the slower the sprint times.

Although NORM is faster than FFS, they do not differ with respect to horizontal peak force, mean force or mean power and are comparable to similar measurements provided by Frost et al. if normalized to body weight [6]. In addition, the force profiles of NORM and FFS were similar, although with a minor early force peak before the absolute force peak in FFS, but shifted in time ( $\mathbf{F i g}$. 3 ). The

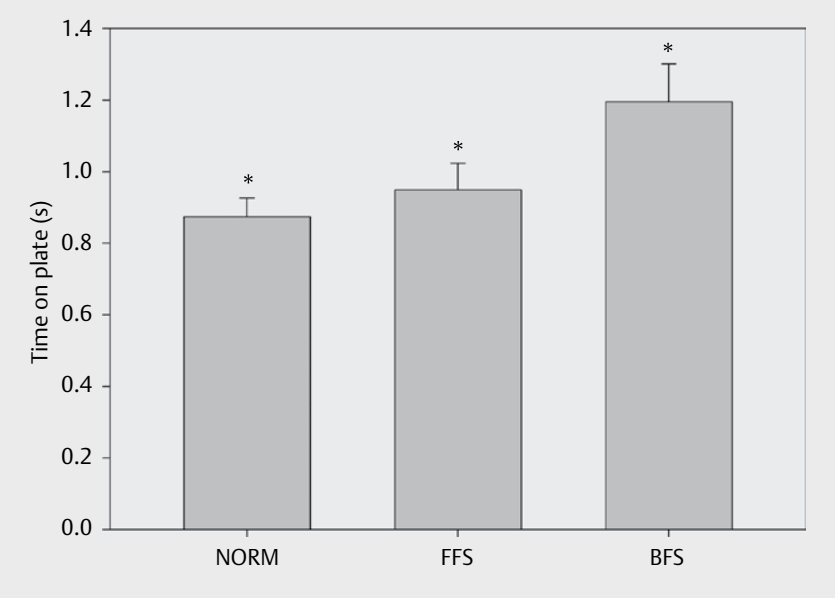

- Fig. 6 Mean time spent on the force plates for each starting technique (MEAN $\pm S D)$. * Significantly different from all other techniques $(\mathrm{p}<0.05)$. 
main difference between these 2 starts is that although NORM is quicker off the start line, the FFS provides greater horizontal momentum after the push-off. There seems to be a rather logical relationship between the time it takes to leave the force plates and the impulse created at this point - the longer you initially are in contact with the ground, the longer you can create horizontal force, enabling you to gain greater initial momentum, as seen with the greater initial momentum created using FFS and BFS compared to NORM. However, because it takes longer to get off the start, the sprint times become longer, as seen with the faster sprint times from FFS compared to BFS with similar horizontal impulses but more time used during push-off to generate this impulse. Similarly, NORM provides the fastest sprint times while using the least time on the push-off creating the least momentum during the get-off. In addition, no differences were found in the peak force, indicating that the SSC has little to no changed effect in increasing the force through the push-off phase of the different techniques. Thus the techniques mainly provide different methods for shifting the centre of mass in front of the point of contact [7].

Because the force plates were stationary, foot placement throughout the push-offs was somewhat locked. As a result, some of the sprint techniques may have been performed in an unnatural manner to ensure contact with the force plates instead of over- or undershooting them. Any unnatural starting conditions may have caused sprint starts to be less than optimal [16]. However, this did not seem to affect the participants, who throughout testing consistently performed their get-off techniques placing their feet on the force plates; therefore, this issue seems to have had little impact on the results.

This study was performed without greater practice of the NORM, FFS and BFS starts. All the participants have a background in American football, where the NORM push-off technique is coached [14] and thus the participants may have had a technical bias to perform better using this technique. Because some techniques have to be learned and do not come naturally, the results may change if a participant is coached in the techniques over a longer time and can perform the push-off without focusing on the technique. However, this study did not include such training prior to testing, and therefore we cannot conclude whether our results are due to superior NORM technique over other techniques or if participants were simply better at NORM from prior training. Further investigation should examine the training effect on sprint performance using the FFS and BFS starting techniques. In addition, further investigations should examine if and how get-off performance changes in the 3 techniques when executed wearing football cleats on a turf or grass field, making the shoe-to-field traction similar to that during practices and matches. A change in traction could shift the result in favour of other push-off techniques.

Because the NORM get-off technique outperforms the BFS and FFS techniques, NORM should be the favoured get-off technique for coaches and players from a stationary staggered stance, as seen in American football, $400 \mathrm{~m}$ sprints and baseball. However, because the advantages of the NORM technique are small, supporting faster $5 \mathrm{~m}$ sprint times only in the magnitude of a few milliseconds, NORM is only feasible for short straight-line sprints, because the advantages of a faster sprint start become diluted by other factors of sprint performance as sprint length increases [7]. Therefore, athletes and coaches should focus mainly on increasing sprint performance through sprint technique and physical training because get-off performance contributes little to longer sprint distance performance.

In conclusion, when including time spent during the get-off, the BFS technique provided the slowest $5 \mathrm{~m}$ sprints, even though earlier studies suggest otherwise [10] due to the inclusion of reaction time and time spent to perform. Thus, athletes using the staggered stance in sprints ought to avoid this technique. Because the NORM start provided the fastest sprint times of the tested techniques, this should be the favoured technique for coaches and for athletes starting from a stationary staggered stance and with the goal of maximising straight-line sprint performance.

\section{Conflict of Interest}

The authors declare that they have no conflict of interest.

\section{References}

[1] Brechue W, Mayhew J, Piper F. Characteristics of sprint performance in college football players. J Strength Cond Res 2010; 234: 1169-1178

[2] Brown T, Vescovi J. Is stepping back really counterproductive? Strength Cond J 2004; 26: 42-44

[3] Coh M, Jost B, Skof B, Tomazin K, Dolenec A. Kinematic and kinetic parameters of the sprint start and start acceleration model of top sprinters. Gymnica 1998; 28: 33-42

[4] Cronin J, Green J, Levin G, Brughelli M, Frost D. Effect of starting stance on initial sprint performance. J Strength Cond Res 2007; 21: 990-992

[5] Cusick J, Lund R, Ficklin T. A comparison of three different start techniques on sprint speed in collegiate linebackers. J Strength Cond Res 2014; 28: 2669-2672

[6] Frost D, Cronin J. Stepping back to improve sprint performance: A kinetic analysis of the first step forwards. J Strength Cond Res 2011; 25: $2721-2728$

[7] Frost D, Cronin J, Levin G. Stepping backward can improve sprint performance over short distances. J Strength Cond Res 2008; 22: 918-922

[8] Hader K, Palazzi D, Buchheit M. Change of direction speed in soccer: How much braking is enough? Kinesiology 2015; 47: 67-74

[9] Harriss DJ, Atkinson G. Ethical standards in sports and exercise science research: 2016 update. Int J Sports Med 2015; 36: 1121-1124

[10] Johnson T, Brown L, Coburn J, Judelson D, Khamoui A, Tran T, Uribe B. Effect of four different starting stances on sprint time in collegiate volleyball players. J Strength Cond Res 2010; 24: 2641-2646

[11] Komi PV. Physiological and biomechanical correlates of muscle function: effects of muscle structure and stretch-shortening cycle on force and speed. Exerc Sport Sci Rev 1984; 12: 81-121

[12] Kraan GA, van Veen J, Snijders C, Storm J. Starting from standing: why step backwards? J Biomech 2001; 34: 211-215 2001

[13] Mero A, Komi PV, Gregor RJ. Biomechanics of sprint running: a review. Sports Med 1992; 13: 376-392

[14] Norvell J. Complete Wide Receiver. Champaign, IL: Human Kinetics; 2013

[15] Pincivero D, Bompa T. A physiological review of American football. Sports Med 1997; 23: 247-260

[16] Slawinski J, Dumas R, Cheze L, Ontano G, Miller C, Mazure-Bonnefoy A. $3 \mathrm{D}$ kinematic of bunched, medium and elongated sprint start. Int J Sports Med 2012; 33: 555-560 